\title{
L'impact des TICE sur la formation des enseignants en Corée
}

The impact of Educational ICT on teacher training in Korea

El impacto de las TICE sobre la formación de los profesores en Corea

Kwanyoung Kim, Jimin Park et Chanhoo Song

Traducteur : Jérôme Quintana

\section{(2) OpenEdition}

\section{Journals}

Édition électronique

URL : http://journals.openedition.org/ries/986

DOI : 10.4000/ries.986

ISSN : 2261-4265

Éditeur

Centre international d'études pédagogiques

Édition imprimée

Date de publication : 1 décembre 2010

Pagination : 129-140

ISBN : 978-2-8542-583-7

ISSN : $1254-4590$

Référence électronique

Kwanyoung Kim, Jimin Park et Chanhoo Song, « L'impact des TICE sur la formation des enseignants en Corée », Revue internationale d'éducation de Sèvres [En ligne], 55 | décembre 2010, mis en ligne le 01 décembre 2012, consulté le 10 décembre 2020. URL : http://journals.openedition.org/ries/986 ; DOI : https://doi.org/10.4000/ries.986 


\section{L’impact des TICE sur la formation des enseignants en Corée*}

\section{Kwanyoung Kim Jimin Park Chanhoo Song}

L'éducation a toujours été l'un des sujets les plus controversés en Corée. De ce fait, toutes les fois qu'un nouveau gouvernement arrive au pouvoir, il s'efforce de répondre aux besoins du pays en offrant une vision de l'éducation qui diffère de celle des précédents gouvernements. Si les politiques éducatives menées par les différents gouvernements n'ont pas mis l'accent sur les mêmes points, elles ont toutefois eu pour point commun de promouvoir la qualité et l'excellence en matière d'éducation. La diversification est une notion rare en Corée. Pour avoir une politique éducative qui accepte et mette en valeur la diversité, il faut d'abord avoir une culture qui accepte et mette en valeur la diversité à la maison, à l'école et dans la société. En outre, afin de favoriser l'amélioration des aptitudes des enseignants et leur diversification, la formation des enseignants à distance a été établie comme mode de formation permettant un apprentissage centré sur l'élève (student-centered learning) et un apprentissage autodirigé ${ }^{1}$ (self-directed learning), en utilisant le cyberespace comme terrain d'apprentissage avec l'aide des technologies de l'information et de la communication (TIC).

Cet article présente la formation des enseignants aux TICE, dans sa première phase (de 1988 à 1996), dans sa deuxième phase (de 1997 à 2000), dans sa troisième phase (de 2001 à 2005), puis dans sa quatrième phase à partir de 2006, selon d'une part les plans mis en place pour les systèmes intégrés de gestion de l'information dans le cadre de la formation des enseignants, et d'autre part selon la performance et l'efficacité de l'action menée. Enfin, l'article conclut sur les perspectives d'avenir en matière de formation des enseignants aux TICE en Corée.

\footnotetext{
* Article traduit par Jérôme Quintana.

1. Processus dans lequel l'apprenant prend en charge les modalités de son apprentissage et en assume l'organisation, la conduite et l'évaluation (NdT).
} 


\section{ÉVOLUTION DE LA FORMATION DES ENSEIGNANTS AUX TICE}

Dès 1988, les établissements scolaires de toute la Corée ont reçu des $\mathrm{PC} / \mathrm{XT}^{2}$ à 16 bits. D’autres outils périphériques liés aux TIC ont commencé à être utilisés dans les écoles grâce aux programmes de formation des enseignants aux TICE pour promouvoir l'utilisation des technologies de l'information et de la communication en milieu scolaire. La formation des enseignants aux TICE, à la fin des années 1980, visait essentiellement à donner les bases de l'informatique, par la compréhension non seulement de la machine, mais également du système d'exploitation et du traitement de texte comme logiciel. Il s'agissait surtout de promouvoir les aptitudes de base des enseignants dans l'utilisation des TICE.

Les programmes de formation des enseignants ont été élaborés aux fins de renforcer la capacité à utiliser l'informatique et les outils périphériques, et d'accroître le nombre d'enseignants en formation en 1995. Des formations plus complètes que celles centrées sur l'acquisition des concepts de base ont été mises en place lorsque des PC à 32 bits ont été distribués dans les établissements scolaires sous le système d'exploitation de Windows et via le réseau Internet. Durant cette période, les programmes de formation mis en place visaient à accroître les aptitudes des enseignants à utiliser les TIC et à favoriser l'utilisation de ces outils dans l'enseignement et l'apprentissage. Au total, 263370 enseignants ont bénéficié d'une formation entre 1988 et 1996.

Pour accroître la capacité des enseignants à utiliser les TIC alors que les classes, grâce à l'introduction de technologies informatiques de pointe, devaient s'adapter rapidement au changement, le ministère coréen de l'Éducation, de la Science et de la Technologie (MEST) a lancé en 1997 le «Plan de promotion des TICE pour les enseignants ", dans le cadre de la formation des enseignants aux TICE. Ce plan visait à former un quart de l'ensemble des enseignants qui devaient participer au programme de formation aux TIC, de sorte que tous puissent bénéficier d'une formation au moins une fois tous les quatre ans. Il a été créé pour permettre d'acquérir des notions d'informatique. Les niveaux suivants avaient pour but de vérifier que les enseignants étaient à même d'effectuer des mises en réseau, d'utiliser des logiciels éducatifs et de produire des supports éducatifs. Au total, 34635 enseignants ont suivi une formation aux TICE entre 1997 et 2000. D’importantes infrastructures avaient été créées pour les écoles primaires, les collèges et les lycées si bien qu'en décembre 2000, tous les établissements scolaires du pays bénéficiaient d'infrastructures pour les TIC : installation de réseaux Internet dans les écoles, classes informatisées, PC pour les enseignants et matériels de pointe pour l'enseignement.

2. Modèle d'ordinateur personnel (PC) lancé par IBM en 1983 (NdT). 
De ce fait, le MEST a créé et présenté le «Plan TICE » comme plan de grande envergure pour réussir à mettre en place des infrastructures de technologies de l'information et de la communication dans chaque salle de classe. Ce plan avait également pour but d'améliorer la qualité des cours par la mise en place de la deuxième phase du plan de formation aux TICE. Dans cette deuxième phase de formation, les enseignants étaient contraints de se former tous les trois ans et le programme de formation aux TICE devait atteindre plus du tiers du nombre total des enseignants. Les programmes de formation ont été modifiés pour se fonder sur les applications et non plus sur les connaissances, afin que le pourcentage de formations aux TICE augmente, passant de 10,8\% en 2001 à $50 \%$ en 2005. Ces modifications dans les programmes de formation ont eu pour conséquence le fait qu'en plus des enseignants des établissements classiques, ce sont aussi les enseignants des établissements spécialisés et autres professionnels de l'éducation qui ont été formés depuis 2001. Selon le KERIS $^{3}$ (2008), environ 1,5 million d'enseignants et de professionnels de l'éducation ont été formés via les programmes que le cadre national de planification et de mise en œuvre a encouragés. Récemment, la création en 2006 d'un Centre agréé assurance qualité de formation en ligne a permis de développer une coopération étroite avec des multinationales de l'industrie des TICE. Parallèlement, un programme de formation des enseignants a été mis en place tout au long de la vie professionnelle des enseignants afin de développer au maximum la formation et de la rendre plus efficace. Au total, entre 2001 et 2008, ce sont 750000 enseignants qui ont reçu une formation en Corée.

\section{TENDANCES DANS LA FORMATION DES ENSEIGNANTS CORÉENS AUX TICE}

\section{Avant l'an 2000}

La formation des enseignants aux TICE reposait sur deux programmes différents de formation avant l'an 2000 ; un programme normal de formation continue des enseignants et un programme spécialisé pour les maîtres enseignants ${ }^{4}$ et les personnels de direction. Pour la formation dite normale, il s'agissait d'initier les enseignants à la compréhension des composantes structurelles et des principes de l'informatique. Le programme, centré sur les connaissances

3. Keris : Korea Education and Research Information Service (Service d'information pour l'éducation et la recherche de Corée).

4. Le ministère coréen de l’Éducation a créé en 2008 cette nouvelle catégorie d'enseignants chevronnés, recrutés sur leur expérience et leurs compétences, et chargés, outre leur service d'enseignement classique, de former et conseiller les nouveaux enseignants, de donner des conférences dans les instituts de formation des enseignants, de mener des recherches sur le terrain, etc. Ils sont recrutés pour une période d'un an et peuvent bénéficier d'une réduction de service de $20 \%$ afin de mener à bien leurs tâches de formation. En mars 2009, ce programme pilote a été étendu à tout le primaire et le secondaire. Source : Korea Institute of Science and Technology Information (KISTI). Pour en savoir plus : http://www.kisti.re.kr/ (NdT). 
en technologies de l'information, visait à améliorer graduellement la capacité à utiliser des traitements de texte, des tableurs, des outils de présentation et des informations obtenues sur Internet. En tout, 604005 enseignants ont reçu une formation entre 1988 et 2000, en moyenne 1,8 fois par personne. De 1997 à 2000, un quart de l'ensemble des enseignants a bénéficié d'une formation aux TICE. Ainsi, chaque enseignant a pu recevoir une formation au moins une fois durant cette période.

Le programme spécialisé, de son côté, a été mis en place à partir de 1992 afin de former les maîtres enseignants aux TICE, y compris les formateurs des bureaux de l'éducation de la métropole et de la province, les experts en matière de contenus éducatifs et les personnels des facultés chargés de la formation en informatique des établissements scolaires. Entre 1992 et 1996, 61 heures de cours de «formation au développement de contenus éducatifs » et de "formation d'experts en apprentissage par l'informatique » ont été mises en place, et la désignation de ces formations a été modifiée entre 1997 et 1998 pour devenir respectivement "formation au développement de didacticiels multimédias » et «formation d'experts en TICE ». Plus tard on a ajouté la «formation de formateur à l'utilisation d'Internet » pour répondre aux besoins des participants.

Les programmes de formation ont été redéfinis à partir de 1999. La «formation au développement de didacticiels multimédias » s'est divisée en «formation au développement de didacticiels multimédias » et en "formation au développement de contenus multimédias ". De la même façon, à partir de 1999 la «formation de formateur à l'utilisation d'Internet » a été divisée en «formation d'instructeur pour l'utilisation d'Internet » et en "formation d'administrateur de réseau ». Au total, 2518 experts en TICE et maîtres enseignants ont effectué chaque formation spécialisée, élaborée pour être en phase avec les nouvelles tendances en éducation et promouvoir l'utilisation des TICE pour une meilleure qualité du service d'éducation (rapport du KERIS sur la cyberformation des enseignants, 1998 ; rapport du KERIS sur la formation des enseignants aux multimédias, 1997).

\section{Après 2001}

Le MEST a annoncé en 2001 un «Plan TICE » pour renforcer l'enseignement public. Ce plan était composé de plusieurs programmes visant au développement des aptitudes des enseignants en matière de TICE, à l'élaboration de contenus éducatifs, à la création de systèmes de soutien pour le cyberenseignement et le cyber-apprentissage, à la promotion de l'utilisation des TICE et à l'amélioration des infrastructures de TIC. Par ailleurs, le « Développement de la maîtrise de l'information par les enseignants » comprenait la formation des enseignants aux TICE, l'utilisation des TIC dans les recherches effectuées pour l'élaboration des cours, l'organisation de concours de recherches en matière 
de TICE, la mise en place d'un certificat de maîtrise de l'information pour les enseignants, la promotion des TICE ainsi que l'élaboration de programmes de formation aux TICE.

La formation des enseignants aux TICE a été promue au rang de «formation obligatoire » et les crédits de formation sont attribués aux enseignants par les bureaux d'éducation à l'échelle régionale, la "formation volontaire » étant gérée par les établissements scolaires. Les inspecteurs relevant des bureaux d'éducation à l'échelle régionale choisissent et décident quelles formations seront mises en place.

Premièrement, la « Formation obligatoire aux TICE » vise à former plus de $33 \%$ du nombre total d'enseignants et à faire acquérir la maîtrise de l'information avec l'utilisation des TIC dans l'enseignement et l'apprentissage. Selon le KERIS (2008), en moyenne $30 \%$ des enseignants (du primaire, de collège, de lycée, d'établissements spécialisés) et des professionnels de l'éducation ont suivi jusqu'au bout la formation obligatoire depuis 2001 jusqu'à ce jour, à l'échelle nationale. Néanmoins, il y a eu quelques disparités selon les régions. La province de Gyeongsangnam a enregistré le plus fort taux (42\%) d'enseignants ayant suivi la formation en 2008.

Deuxièmement, la formation des chefs d'établissement vise à former tous les ans $33 \%$ des directeurs d'école, sous-directeurs et professionnels de l'éducation. Les programmes sont élaborés pour encourager les chefs d'établissement à améliorer leurs résultats, par exemple en favorisant le développement des aptitudes à utiliser les TICE, la conception de cours utilisant les TIC, la gestion des établissements par les TIC, la construction d'une communauté d'apprentissage au plan local par le biais du site internet d'un établissement et la comparaison des pratiques nationales et internationales en matière de TICE. Les programmes de formation pour les chefs d'établissement, en termes de nombre de personnels de direction formés, ont connu un résultat remarquable. En 2001, près de $60 \%$ des chefs d'établissement ont suivi la formation à l'échelle nationale (KERIS, 2008). Ce pourcentage a continué d'être élevé jusqu'en 2005, puis a enregistré une baisse pour atteindre moins de $20 \%$ en 2008. En bref, les données indiquent qu'environ la moitié des chefs d'établissement en Corée ont suivi la formation entre 2001 et 2008.

Troisièmement, les programmes de formation aux TICE à l'intention des maîtres enseignants ou des formateurs visant à former des " chefs de file ", capables de donner une impulsion à l'utilisation des technologies de l'information et de la communication appliqués à l'éducation, sont organisés sous l'égide du KERIS. Le nombre de maîtres enseignants formés par le programme entre 2001 et 2008 s'élevait approximativement à plus de 2100 (KERIS, 2008). Près de mille maîtres enseignants du programme de formation aux TICE ont contribué à favoriser le programme de formation des enseignants en Corée. 
Quatrièmement, chaque établissement a élaboré un programme de formation volontaire aux TICE et mis en œuvre ce programme de plus de quinze heures annuelles à inscrire dans le projet d'établissement. Il a été conçu pour encourager l'entraide en favorisant la recherche d'information pour l'élaboration des cours et en promouvant les activités d'experts ainsi que de personnels spécialisés dans l'apprentissage dans l'utilisation des TICE. Les programmes volontaires ont formé plus de 350000 enseignants à l'échelle nationale entre 2001 et 2008 selon le KERIS (2008). Les données révèlent néanmoins de grandes disparités selon les régions. Par exemple, Séoul, la capitale, a enregistré un taux avoisinant les $100 \%$ d'enseignants ayant suivi la formation volontaire. En revanche, Daejeon, Ulsan, la province de Chungcheongnam, la province de Jeollabuk et Jeju n'ont pas mis en place les programmes de formation volontaire avant 2008 .

Tenant compte de la durée de vie professionnelle des enseignants et de leurs compétences, le programme de formation a été défini depuis 2006 selon le schéma suivant :

\section{Système de formation des enseignants aux TICE} (de l'entrée en fonction au départ à la retraite)

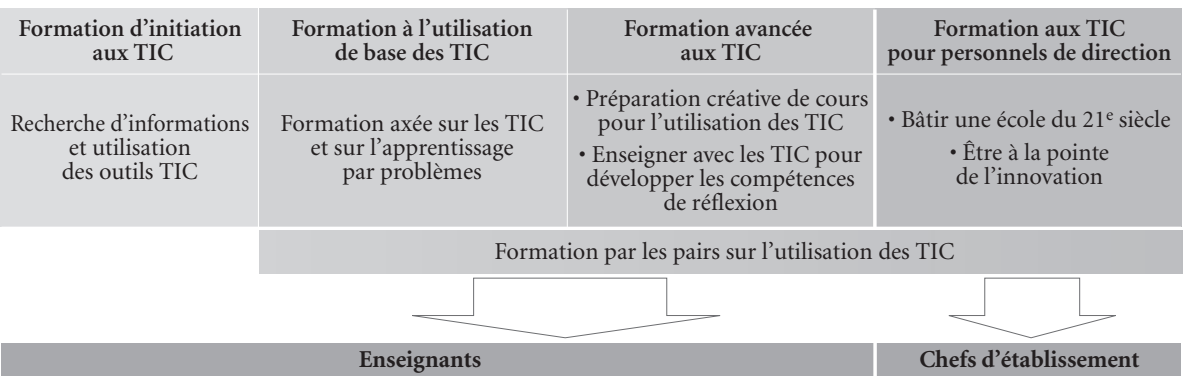

Le programme de formation a été conçu et mis en place afin d'introduire de nouvelles formations et de nouvelles évolutions technologiques. Il a également été adapté aux changements intervenus dans l'environnement éducatif en élaborant de nouveaux programmes de formation pour chaque secteur. Ces nouveaux programmes ont tous été élaborés pour promouvoir le développement d'aptitudes en ciblant tous les acteurs des établissements scolaires, depuis les enseignants jusqu'aux chefs d'établissement. Le programme se divise en quatre champs distincts.

Premièrement, le « programme de formation aux TICE pour les personnels de direction " élaboré pour les chefs d'établissement, sous-chefs et autres professionnels de l'éducation et étendu aux villes et aux provinces en 2006. 
Il est composé de deux parcours de formation : "bâtir une école du $21^{\mathrm{e}}$ siècle » et "gérer/diriger de façon innovante ».

Deuxièmement, le "programme de formation avancée aux TICE » visait les enseignants du primaire et du secondaire (collège) ou ceux qui avaient suivi la formation "École du futur ». Les formations dispensées avaient pour objectif de concevoir des cours créatifs et d'encourager la réflexion critique en utilisant les TIC. Plus de 45000 enseignants ont suivi la formation intitulée « concevoir des cours créatifs en utilisant les TIC » entre 2003 et 2007.

Troisièmement, le «programme de formation à l'utilisation de base des TICE », qui se subdivisait en quatre formations ( utilisation des TIC pour l'apprentissage par problèmes $(\mathrm{APP})^{5}$ », "École du futur », «École du futur (formation actualisée) » et "démarrer». Ce programme a été conçu pour les enseignants du primaire et du secondaire (collège) entre 2002 et 2008. Le programme "École du futur» a été actualisé (en y intégrant "l'apprentissage mixte» par les TIC) en 2008 et mis en place en 2009.

Et enfin, quatrièmement, la « formation entre pairs à l'utilisation des TICE » a été conçue en 2006 pour les animateurs de pointe d'ateliers TIC et les enseignants de chaque école. Près de 700 enseignants ont suivi cette formation en 2007.

Avec la mise en place de l'Apprentissage 2.0, ce nouvel outil d'enseignement-apprentissage fondé sur la participation, l'ouverture et le partage en incluant les changements intervenus dans l'utilisation des TIC, il a fallu élaborer des programmes de formation des enseignants qui permettent le développement de stratégies efficaces pour promouvoir des infrastructures éducatives à même d'intégrer et d'appliquer les nouvelles TICE. C'est ainsi qu'on élabore en 2008 le "programme de formation des enseignants de la nouvelle génération " comprenant la «formation des enseignants pour l'apprenant du $21^{\mathrm{e}}$ siècle (à l'école primaire) » et la formation « démarrer». Le "programme de formation des enseignants de la nouvelle génération » vise à améliorer les compétences des enseignants dans l'adoption de nouvelles technologies appliquées à l'éducation et à développer les nouvelles compétences pédagogiques qui constitueront le nouvel outil d'enseignement-apprentissage, ce qui représente un changement radical dans le domaine de l'éducation.

Afin d'améliorer la compétitivité des enseignants avec le nouveau programme de formation, le "plan pour le système intégré de gestion de l'information dans le cadre de la formation des enseignants » a été mis en place en 2008.

5. APP : apprentissage par problèmes. En anglais, PBL (Problem-based learning). Dans l'apprentissage par problèmes, les apprenants, regroupés par équipes, travaillent ensemble à résoudre un problème généralement proposé par l'enseignant, problème pour lequel ils n'ont reçu aucune formation particulière, de façon à faire des apprentissages de contenu et de savoir-faire, à découvrir des notions nouvelles de façon active (il s'instruit lui-même) en y étant poussé par les nécessités du problème soumis. La tâche de l'équipe est habituellement d'expliquer les phénomènes sous-jacents au problème et de tenter de le résoudre dans un processus non linéaire. La démarche est guidée par l'enseignant qui joue un rôle de facilitateur. Ceci est différent d'une étude de cas ou d'une résolution, en ce sens que l'on ne donne pas forcément de solution. (NdT, d'après Wikipédia). 
Il avait pour but de fournir de façon efficace aux écoles toute information pertinente sur les formations du secteur public organisées par les instances éducatives des villes et des provinces et sur les formations du secteur privé des entreprises, et d'améliorer la qualité du programme de formation en analysant les données statistiques générées et obtenues via le plan. Le "système renforcé de gestion de l'information dans le cadre de la formation des enseignants » a été élaboré par étapes pour développer des infrastructures permettant de recueillir l'information. Ce système gère le programme de formation des enseignants et introduit un système d'auto-évaluation des enseignants avec possibilité de consulter des conseillers depuis 2009, comme indiqué dans la figure ci-dessous.

\section{Plan pour le système intégré de gestion de l'information dans le cadre de la formation des enseignants}

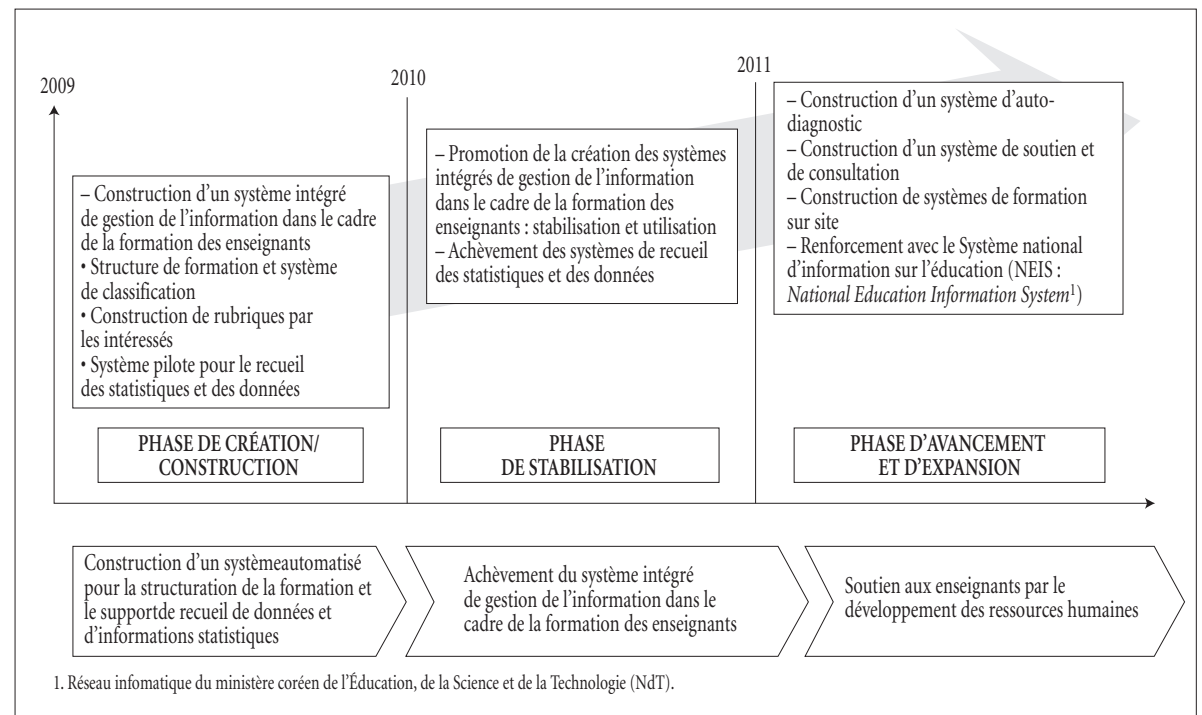

\section{Perspectives d’aveniR}

La formation des enseignants aux TICE est parvenue à améliorer les compétences pédagogiques en matière d'utilisation des TIC et à transformer le rôle des enseignants dans cette nouvelle ère de l'éducation. De fait, le programme de formation des enseignants a dû relever le défi de se renouveler alors que des changements radicaux intervenaient dans le domaine de l'éducation. Quatre perspectives se dégagent quant à l'avenir de la formation des enseignants aux TICE.

Premièrement, la formation des enseignants a dû répondre aux changements rapides qui sont intervenus dans l'environnement éducatif pour suivre le mouvement de l'époque. Le projet Web 2.0, qui incarne l'esprit de "participation ", de "partage » et "d'ouverture », a entraîné des changements radicaux 
dans la société et il a nécessairement conduit à mettre en place des réformes innovantes du modèle de l'enseignement et de l'apprentissage. Ainsi, la formation des enseignants est nécessaire pour répondre à ces besoins de nouvelles orientations de l'éducation et s'efforcera de proposer des programmes de formation optimaux. De nouvelles formations ont été élaborées, visant à fournir des connaissances essentielles pour l'élaboration de cours axés sur l'apprenant et pour le renforcement de l'éthique professionnelle en matière d'information et de communication.

Deuxièmement, il est important de reconnaître l'importance des TICE comme modèle fondamental pour définir les contours de l'éducation de demain. Pour encourager l'utilisation des TICE, il faut inciter les directeurs d'établissement et les personnels d'éducation non seulement à améliorer leur capacité à diriger mais surtout à mener des réformes éducatives innovantes et à opérer des changements éducatifs tout aussi innovants dans les établissements scolaires. C'est pourquoi les programmes de formation à destination des directeurs d'établissement et des personnels d'éducation devraient être élaborés pour favoriser le développement des aptitudes des enseignants et pour soutenir les efforts constants réalisés afin d'améliorer l'environnement éducatif.

Troisièmement, la formation des enseignants, liée au modèle d'enseignement-apprentissage dans le cadre de la stratégie du renouveau de la formation, devrait être améliorée pour inclure non seulement la formation continue mais également la formation initiale. La formation avancée peut aider les enseignants à appréhender plus facilement le nouvel environnement éducatif et à acquérir le modèle d'enseignement-apprentissage de la nouvelle génération. Il est également recommandé d'établir des ponts entre la formation continue et la formation initiale car cela peut créer une synergie et favoriser le développement professionnel.

Quatrièmement, il est possible aux enseignants de mettre en place des stratégies de développement des aptitudes, en renforçant la coopération avec les enseignants d'autres pays. On a souvent demandé à la Corée d'accueillir des formations internationales de développement des aptitudes, avec pour objectif de rendre les enseignants compétitifs à l'échelle mondiale. Il est temps aujourd'hui d'organiser ce type de formation transnationale. Par ailleurs, nous devons garder à l'esprit que nous pouvons mettre en place dans d'autres pays notre programme de formation interne à la Corée.

\section{BIBLIOGRAPHIE}

AHN Mi-Ri (2000) : «Develop the Model for Distance Teacher Training Center and Distance Graduate Teacher Schools and Management ». Korea Association for Educational Information and Media 6(1): 133-167 (Développer le modèle des centres de formation des enseignants à distance et des instituts supérieurs de formation à distance des enseignants et personnel de direction). 
BAK Young-Kyun (1999): Web-based Distance Learning for Teacher Training. Webbased Learning. Kyoyookbook Publish: Seoul. (Formation des enseignants à distance par le Web. Apprentissage en ligne).

BANG Myung-Sook et SHIN Soo-Bum (2001) : Distance Education for Teacher Training Institute Management. Korea Education and Research Information Service. (Formation à distance des enseignants).

BANG Myung-Sook (1997) : Systematic Analysis of Teacher Training for IT Education. Korea Multimedia Education Center. (Analyse systématique de la formation des enseignants aux technologies de l'information).

CHOI Sang Geun (1995): A Study on the teachers intensive-training systems through distance education in Korea. Korea Educational Development Institute. (Étude sur la formation intensive des enseignants à distance en Corée).

Cyber teacher training Report (1998) : Korea Education and Research Information Service and Ministry of Education. (Rapport sur la cyberformation des enseignants).

KIM Hae-Sook (2003) : A Study on Management System and Legal Framework for Teacher Training. Ministry of Education and Human Resources Development. (Étude du système de gestion et du cadre juridique de la formation des enseignants).

KIM Jung-Rang (2001) : A Study on Digitalization of Primary Teacher Training Course. Ministry of Education and Human Resources Development. (Étude sur la numérisation de la formation des enseignants du primaire).

KIM Young-AE (2004) : Evaluation on Distance Learning Education 2004. Korea Education and Research Information Service. (Évaluation de l'apprentissage à distance).

Korea Education and Research Information Service (2008) : Adapting ICT into Education and its Effectiveness: White Paper and Annual Report. (L'adaptation des TIC à l'enseignement et son efficacité).

LEE Byung Jin (1996) : "A Study on the Systems of Inservice Education by Teacher Career Cycles in Korea ». Journal of education research 34 (1):315-345. (Étude des systèmes de formation continue par cycles de carrière enseignante en Corée).

Ministry of Education and Human Resources Development and Korea Education Research and Information Service (2004): The Strategy for Teacher Training Course and Working Process Improvement. (Stratégie de formation des enseignants et amélioration du processus de travail)?

Multimedia Teacher Training Report (1997) : Korea Education and Research Information Service and Ministry of Education. (Rapport sur la formation des enseignants aux multimédias). 